\title{
Methylphenidate treatment of adult male prison inmates with attention-deficit hyperactivity disorder: randomised double-blind placebo-controlled trial with open-label extension
}

Ylva Ginsberg and Nils Lindefors

\section{Background}

Attention-deficit hyperactivity disorder (ADHD) is highly prevalent in prison inmates, but pharmacological treatment has not yet been evaluated in this group.

\section{Aims \\ To evaluate osmotic-release oral system (OROS) methylphenidate in adult male long-term prison inmates with ADHD.}

\section{Method}

Randomised, double-blind, placebo-controlled 5-week trial, followed by 47 -week open-label extension in 30 prison inmates with ADHD and comorbid disorders. Primary outcome was level of ADHD symptoms after 5 weeks, evaluated by a masked assessor. Secondary outcomes were self-reported ADHD symptoms, global severity and global functioning throughout the 52-week trial, and post hoc treatment response and numbers needed to treat (NNT) (trial registration: NCT00482313.)

\section{Results}

Treatment significantly improved ADHD during the trial $(P<0.001$; Cohen's $d=2.17)$, with reduced symptom severity and improved global functioning. The placebo response, cardiovascular measures and adverse events were non-significant; the NNT was 1.1. Attention-deficit hyperactivity disorder symptoms, global severity and global functioning continued to improve during the open-label extension.

\section{Conclusions}

Osmotic-release oral system methylphenidate is an effective treatment for adult male prison inmates with ADHD.

\section{Declaration of interest}

Y.G. has served as an investigator for Janssen-Cilag, and as a consultant and speaker for Janssen-Cilag and Novartis.
Attention-deficit hyperactivity disorder (ADHD) is a common, inherited and disabling developmental disorder with early onset. Symptoms of ADHD often persist into adulthood. A recent meta-analysis of population-based studies across several countries estimated the pooled prevalence of adult ADHD to be 2.5\% (95\% CI 2.1-3.1). ${ }^{1}$ However, most of the populations in these studies were unbalanced with regard to gender and age, and applied different tools to diagnose ADHD according to DSM-IV. ${ }^{2}$ Therefore, the reported prevalence rates may not reflect the general population across these countries. Attention-deficit hyperactivity disorder is associated with pervasive cognitive, emotional and functional impairments that affect various aspects of life, including educational, occupational and social performance. ${ }^{3,4}$ In addition, nearly $80 \%$ of adults with ADHD present with at least one comorbid disorder, particularly anxiety disorders, affective disorders, substance use disorder and antisocial personality disorder. ${ }^{5,6}$ Both substance use disorder and antisocial personality disorder increase the risk for delinquency, and approximately $25-45 \%$ of adult prison inmates are estimated to have ADHD. ${ }^{7-10}$ Inmates with ADHD have an increased incidence of behavioural problems and comorbid psychiatric disorders, including substance use disorder and personality disorder, compared with other inmates. ${ }^{10-12}$ According to UK guidelines issued by the National Institute for Health and Clinical Excellence (NICE), drugs are the first-line treatment for ADHD in adults with moderate or severe levels of impairment, and methylphenidate is the drug of first choice. ${ }^{13}$ Clinical trials evaluating methylphenidate and other stimulants display short-term efficacy in both children and adults with ADHD. ${ }^{14,15}$ These trials have typically excluded participants with comorbid disorders. The small number of studies evaluating methylphenidate for participants with ADHD and comorbid substance use disorder has not been able to establish efficacy against ADHD. ${ }^{16}$ The long-term effectiveness, safety and tolerability of methylphenidate is not well understood as there have been few long-time follow-up studies. ${ }^{16-19}$ Despite the high prevalence of ADHD in prison inmates, pharmacological treatment has not been evaluated in this group. This is likely due to concerns about safety and misuse of the drug therapies by this patient population, and the challenge of conducting pharmacological trials in prison settings. ${ }^{7-10}$

The purpose of the present study was to evaluate the efficacy, long-term effectiveness, safety and tolerability of osmotic-release oral system (OROS) methylphenidate in adult long-term prison inmates with ADHD and comorbid disorders. The OROS methylphenidate was delivered at a daily dose of $72 \mathrm{mg}$ compared with placebo over a 5-week period, followed by an open-label extension with OROS methylphenidate delivered at a flexible dosage of up to $1.3 \mathrm{mg} / \mathrm{kg}$ daily over a 47 -week period.

\section{Method}

\section{Participants}

Eligible participants were adult male prison inmates, aged 21-61 years, with ADHD according to DSM-IV criteria. ${ }^{2}$ All inmates were hosted at Norrtälje Prison, a high-security prison outside Stockholm, Sweden, for long-term, adult male inmates, typically convicted of violent or drug-related crimes. The initial screening survey and diagnostic assessments have been previously reported. ${ }^{8}$ Briefly, inmates hosted at Norrtälje Prison between December 2006 and April 2009 were approached for screening for both 
childhood and adulthood ADHD by self-reported questionnaires. All inmates were approached except those deemed too mentally unstable and those to be deported from Sweden after serving their sentence. The aim was to recruit 30 eligible participants with established ADHD to the randomised clinical trial (RCT): participants were initially selected on the basis of the ADHD questionnaires, with diagnosis subsequently confirmed in comprehensive assessments by experienced board-certified psychiatrists and clinical psychologists. Participants were mainly recruited from Stockholm County, and had at least 14 months left until conditional release to ensure completion of the trial. The study was approved by the Ethical Board of Stockholm, Sweden (2006/1141-31/3) and the Swedish Medical Products Agency (EudraCT-nr 2006-002553-80). The trial is registered with clinicaltrials.gov (NCT00482313). Written informed consent was obtained from the participants after they had received a thorough description of the study. To validate adherence to good clinical practice and the Declaration of Helsinki, the trial was independently monitored by the Karolinska Trial Alliance and inspected by the Swedish Medical Products Agency respectively.

\section{Clinical assessments}

The assessments included the Structured Clinical Interview for DSM-IV Axis I Disorders (SCID-I), ${ }^{20}$ the Hare Psychopathy Checklist - Revised (PCL-R), ${ }^{21}$ a self-rated version of the Structured Clinical Interview for DSM-IV Axis II Personality Disorders, the SCID II Patient Questionnaire (SCID-II-PQ), ${ }^{20}$ and a structured interview confirming ADHD consistent with DSM-IV. ${ }^{2}$ Additional assessments included a medical history, a physical examination, routine laboratory tests, urinary drug screening and neuropsychological tests assessing IQ and executive functions. When appropriate, additional evaluations were performed for autism-spectrum disorder in accordance with DSM-IV. ${ }^{2}$ Finally, the assessments included obtainment of information from parents by questionnaires and interviews, and collection of records from school, health services, and the prison and probation service, regarding childhood history and present symptoms and functioning.

\section{Inclusion and exclusion criteria}

To enter the trial, participants had to have confirmed ADHD in accordance with DSM-IV and to agree not to behave violently during the study. Participants with comorbid disorders such as autism-spectrum disorder, anxiety and depression could take part if they were considered to be stable at baseline. Previous drugelicited episodes of psychosis were not a cause for exclusion, other than chronic psychoses. Concurrent medication not interfering with methylphenidate was permitted for treating comorbid disorders, as long as doses were stable for at least 1 month at baseline. Medications interfering with methylphenidate had to be tapered off before the baseline visit took place. Participants were excluded if they were known to be non- responsive or intolerant to methylphenidate, or intolerant to lactose. In addition, participants were excluded if they showed evidence of substance misuse up to 3 months before baseline, assessed in urine samples. Intellectual disability, epilepsy, glaucoma, uncontrolled hypertension, angina pectoris, cardiac arrhythmias, cardiac abnormality or a family history of serious cardiac illnesses were exclusion criteria, but hepatitis $\mathrm{C}$ without liver insufficiency did not preclude inclusion.

\section{Randomisation and masking}

Participants were randomly assigned in equal numbers to either the placebo or the OROS methylphenidate group according to a parallel-group design. The pharmacy laboratory assigned participants to the two study groups using a random number table prior to preparing and dispensing the study drug according to the study protocol. The random number table was stored in the pharmacy department and was concealed from study staff and participants until completion of the study. The placebo and methylphenidate capsules and packaging were identical in appearance and were coded with a unique randomisation number.

\section{Intervention}

Both study staff and participants were masked to assignment during the RCT. Participants were consecutively randomised in the order that they were enrolled by the investigator. The study drug was titrated from $36 \mathrm{mg} /$ day for 4 days to $54 \mathrm{mg} /$ day for 3 days and then to $72 \mathrm{mg} /$ day for the remaining 4 weeks. All participants completing the 5-week RCT were eligible to enter the 47-week open-label extension, starting the day after completion of the 5-week RCT. During the open-label extension, methylphenidate was titrated from $36 \mathrm{mg} /$ day to determine the optimal response and tolerability, but not exceeding $1.3 \mathrm{mg} / \mathrm{kg}$ daily. In case of adverse events, downward titration was allowed, followed by upward titration once the participant had recovered. In addition to medication, all participants received personal psychosocial treatment as part of prison routine, including school activities according to the Swedish curriculum and cognitive programmes addressing addiction, criminality, aggression and social skills. The psychosocial treatment did not specifically address ADHD.

\section{Primary outcome measure}

The primary efficacy outcome was the change in ADHD symptoms from baseline until completion of the RCT at end of week 5, as measured by the total sum-score of the Conners' Adult ADHD Rating Scale - Observer: Screening Version (CAARS-O:SV), ${ }^{22}$ which is one of the most widely used expert rating scales in clinical trials evaluating adult ADHD. This strictly DSM-IV-oriented 18-item Likert scale includes relevant psychometric properties based on US norms. Although it has been widely used internationally, including Sweden, its use has not been formally evaluated in non-English speaking countries. $^{22}$

\section{Secondary outcome measures}

The frequency of adult ADHD symptoms corresponding to DSM-IV criteria were reported by participants using the Adult ADHD Self-Report Scale (ASRS). ${ }^{22}$ This 18-item Likert scale includes relevant psychometric properties based on US norms. The ASRS also lacks Swedish validation, but is widely used in clinical practice. Symptoms of ADHD during the open-label extension were evaluated by the masked assessor using CAARS-O:SV. Also, overall severity from ADHD using the Clinical Global Impression Severity Scale (CGI-S ${ }^{23}$ and psychosocial functioning using the Global Assessment of Functioning Scale (GAF), ${ }^{24}$ were evaluated by the same assessor, from baseline until end of week 52 .

Treatment response rate and numbers needed to treat (NNT) were determined post hoc.

Changes in neuropsychological functioning, quality of life and institutional behaviour during the course of the study were also evaluated; these findings will be reported in a subsequent paper. 


\section{Procedures}

The screening visit took place up to 2 weeks before randomisation to enable medications excluded from the study to be tapered off prior to the baseline visit. In most cases, the same specifically trained and masked assessor performed the evaluations of CAARS-O:SV, CGI-S and GAF at baseline and weeks 1, 3, 5, 8, 16, 32 and 52. The self-reported ASRS was also administered at these visits. Body weight was recorded at baseline and weeks 5 , $8,12,20,32,44$ and 52. Adverse events, blood pressure and heart rate were obtained at all study visits. In addition, prison staff regularly supervised urinary drug screenings as part of general prison routines. To certify adherence to the protocol, study staff supervised and documented delivery of the study drug. All packages, documentation and remaining drugs were returned to the primary investigator at the end of the study for evaluation of adherence to the study protocol.

\section{Statistical analysis}

Descriptive statistics summarised demographics, clinical characteristics and outcome scores at baseline. Paired $t$-tests were performed for changes within participants over time and mixed between-within participants ANOVA for changes from baseline through to the final visit of the RCT, using SPSS 17.0 for Windows for both outcome and safety measures, including cardiovascular measures and body weight. All analyses were intention-to-treat (ITT) using last observation carried forward (LOCF), including all randomised participants, with two-sided significance set at $P=0.05$. Non- parametric statistics were used for outcomes measured by Likert scales. As results for parametric and nonparametric tests were similar, only parametric statistics are presented. Single missing values were handled conservatively by substituting the missing value with the higher value from the preceding or following visit. The effect size was analysed using Cohen's $d$ for efficacy measures. Post hoc, the treatment response rate and NNT for treatment response were determined.

\section{Sample size}

Based on preliminary findings and previous studies, ${ }^{14,25,26}$ the aim was to detect a mean difference of 9 units in the CAARS-O:SV (range 0-54) between groups from baseline until the end-point at week 5. Power was 90\%, significance level was 0.05 (two-sided) and s.d. $=7$. With treatment assignment at a 1:1 ratio and allowing for a $20 \%$ drop-out rate, the aim was to recruit 30 participants.

\section{Results}

\section{Participants}

Of 34 assessed inmates, 9 had previously been diagnosed with ADHD, including 2 during childhood. Five inmates had previously been treated with methylphenidate, one of them during childhood, for no more than a few months. None of the inmates were known to be non-responsive or intolerant to methylphenidate.

Clinical assessments confirmed that 30 of the inmates had ADHD. All consented and were randomly assigned at a 1:1 ratio to placebo or OROS methylphenidate $72 \mathrm{mg}$ daily, between May 2007 and April 2009 (Fig. 1). Most participants were initially hosted in a dedicated ADHD wing for 12 inmates, as decided by Norrtälje Prison. This wing was separate from other wings to prevent exchange of drugs. The prison officers in the wing were educated by the investigators about ADHD. Inmates were relocated to ordinary prison wings once their participation in the trial was completed. A small number of participants carried out the trial in other special wings, such as the wing for sexual offenders.
Baseline characteristics were largely similar between study groups, as shown in online Table DS1. Comorbid disorders were common; all reported lifetime substance use disorder, all but one presented antisocial personality disorder, a majority had mood and anxiety disorders, and a quarter had confirmed autism-spectrum disorder. At baseline, almost half of the participants were receiving concurrent treatment for mood and anxiety disorders. Baseline scores on CAARS-O:SV, ASRS, CGI-S and GAF show that the participants had severe ADHD and low psychosocial functioning at entry.

\section{Intervention}

All participants received the assigned treatment and completed the 5-week RCT; no one dropped out. All 30 participants subsequently entered the 47-week open-label extension, ending in April 2010. The open-label extension was completed by 24 participants. However, 25 participants provided end-point data for the open-label extension as we assessed one participant at week 46 , just prior to advanced release from prison. Figure 1 presents time and reasons for discontinuation. Participants who first received placebo treatment were more likely to drop out compared with those who initially received OROS methylphenidate. The ITT and safety analyses using LOCF included all 30 participants. Treatment adherence was excellent; 99\% for the RCT and $98 \%$ for the open-label extension. However, symptom relief did not last long enough for a few participants with once daily delivery. Therefore, we modified the study protocol to permit delivery of OROS methylphenidate twice daily during the open-label extension, in the morning and at noon, to maintain symptom relief throughout the day. At end-point week 52, the mean dose was $105 \mathrm{mg}$ daily (s.d. $=27.2)$ or $1.22 \mathrm{mg} / \mathrm{kg}$ daily (s.d. $=0.28)$.

\section{Primary outcome measure}

Mean scores for the primary outcome measure, CAARS-O:SV, were significantly decreased by 19.6 (95\% CI 14.7 to 24.5$)$ in the methylphenidate group $(P<0.001)$ compared with a nonsignificant decrease of 1.9 ( $95 \%$ CI -0.4 to 4.2 ) in the placebo group. The effect size was exceptionally large, with a Cohen's $d$ score of 2.17 (online Table DS2 and Fig. 2).

\section{Secondary outcome measures}

Change in CAARS-O:SV was also measured from the completion of the RCT (week 5) to the end of the open-label extension (week 52). Scores for CAARS-O:SV improved in both groups over the course of the open-label extension; those who switched from placebo to OROS methylphenidate after 5 weeks improved more than those who received OROS methylphenidate for the full 52 weeks (online Table DS2 and Fig. 2). Over the entire 52-week period, participants who received placebo during the first 5 weeks did not improve as much as those who received OROS methylphenidate from the start (online Table DS2 and Fig. 2).

Other secondary outcomes were changes in ASRS, CGI-S and GAF during both the RCT and the open-label extension. As shown in online Table DS2, the OROS methylphenidate group significantly outperformed placebo on all outcome measures during the RCT (ASRS, $P=0.003$; CGI-S, $P<0.001$; and GAF, $P=0.004)$. All outcome measures also improved in both groups during the open-label extension. In summary, the OROS methylphenidate group improved considerably during both phases, whereas the improvement of the placebo group was insignificant during the RCT but was substantial during the open-label extension. Over the entire 52-week study, those who received 


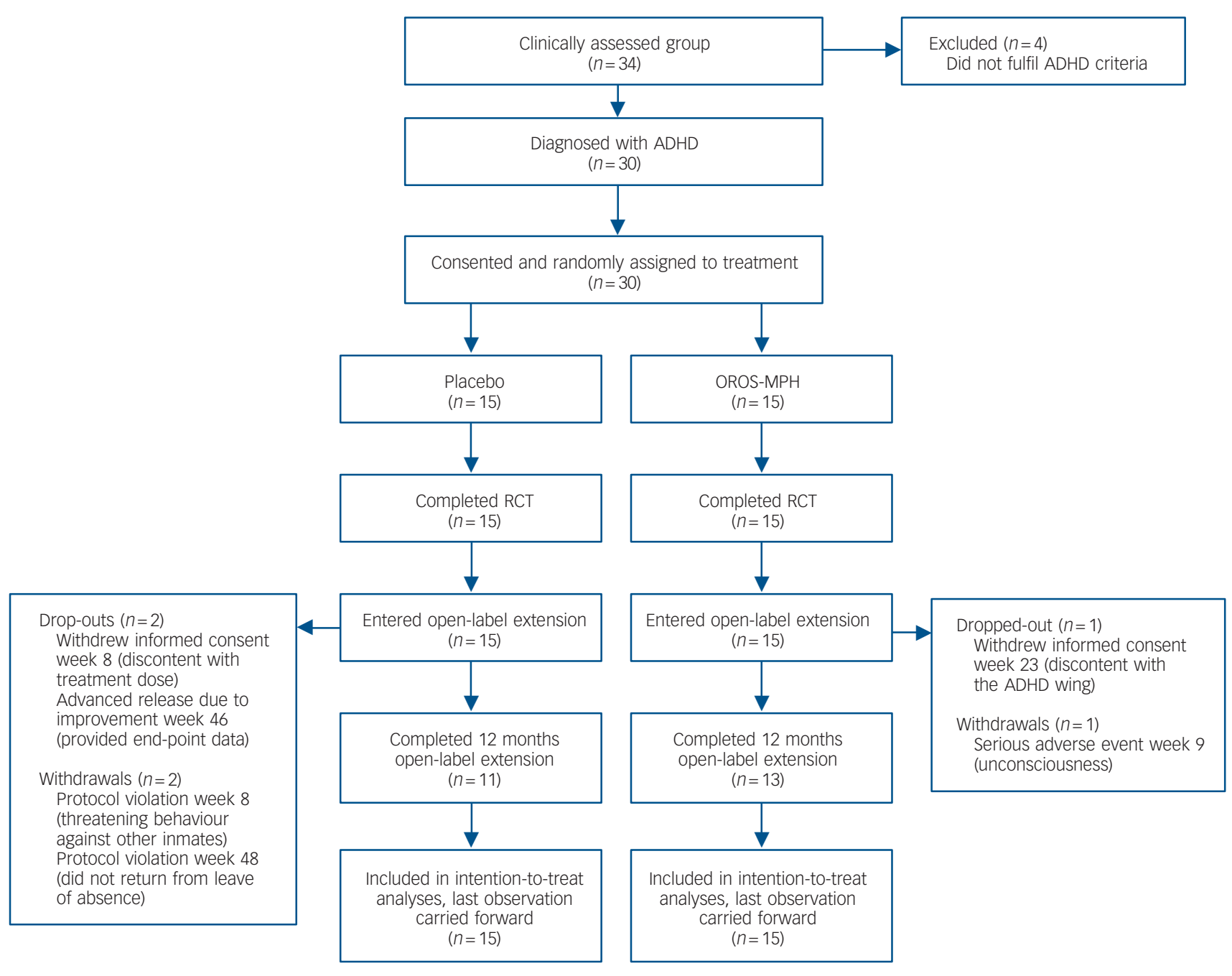

Fig. 1 Participant flow and reasons for dropping out throughout the trial. ADHD, attention-deficit hyperactivity disorder; RCT, randomised controlled trial.

OROS methylphenidate from the start improved the most (online Table DS2).

Post hoc, when applying the original definition of treatment response ( $\geqslant 30 \%$ decrease of CAARS-O:SV at week 5), $87 \%$ responded to OROS methylphenidate treatment compared with $0 \%$ for placebo; NNT was 1.1 (95\% CI 1-2). The extent of response in individual participants is shown in Table 1, with marked responses observed in the methylphenidate group.

\section{Adverse events}

Adverse events occurred more frequently in the methylphenidate group than in the placebo group during the RCT. However, mucosal dryness was the only adverse event that significantly differed between study groups, being more frequently reported in the OROS methylphenidate group. No serious adverse event was recorded during the RCT (data not shown). There was one discontinuation during the open-label extension due to a serious adverse event (Fig. 1); one participant became unconscious at week 9 and was sent to hospital, where he recovered spontaneously. As we could not identify the underlying cause, we immediately stopped medication and withdrew the participant from the trial. The most common adverse events associated with the study drug were abdominal discomfort, headache, mucosal dryness, depressed mood, loss of appetite, anxiety, diarrhoea, sweating, interrupted sleep and fatigue. The intensity of these adverse events was generally mild to moderate and they were not considered to justify removal from the study (data not shown). Regular supervised urinary drug screening did not reveal any drug misuse during the course of the study.

\section{Safety measures}

There were no significant changes in systolic or diastolic blood pressure, heart rate or body weight in either group during the RCT. Over the entire 52-week study period, systolic blood pressure in the methylphenidate group increased by $21.5 \mathrm{mmHg}$ (95\% CI 8.9-34.0) and diastolic blood pressure increased by $11.0 \mathrm{mmHg}$ (95\% CI 4.9-17.1). There were no changes in heart rate or body weight between study groups. Heart rate increased by 13.2 beats per minute (95\% CI 7.0-19.4) from baseline until week 52 in the group that initially received the placebo; body weight, systolic and diastolic blood pressure remained unchanged (online Table DS3).

\section{Discussion}

\section{Main findings}

In this study, OROS methylphenidate was significantly better than placebo in reducing symptoms of ADHD in adult prison inmates with ADHD and comorbid disorders, including lifetime substance use disorder, in a 5-week RCT. The therapeutic effect of OROS methylphenidate continued to improve during the 47-week 
Table 1 Treatment response among participants in both

study groups during the 5 -week randomised controlled trial

$\begin{array}{ccc}\text { Treatment response, \% }{ }^{\mathrm{a}} & \text { OROS-MPH } & \text { Placebo } \\ (n=15) & (n=15)\end{array}$

\begin{tabular}{|lcc|}
\hline None $(\leqslant 0)$ & 1 & 2 \\
\hline Small $(1-25)$ & 1 & 13 \\
\hline Moderate $(26-50)$ & 6 & 0 \\
\hline High $(51-75)$ & 7 & 0
\end{tabular}

OROS-MPH, osmotic-release oral system methylphenidate.
a. Treatment response defined as \% decrease of the total sum-score of Conners

a. Treatment response defined as \% decrease of the total sum-score of Conners'
Adult ADHD Rating Scale - Observer: Screening Version from baseline until end of th Adult ADHD Rating
trial at week 5.

open-label extension. The treatment was safe and well-tolerated with the exception of one serious adverse event, and despite a high prevalence of lifetime substance use disorder, no drug misuse was detected during the course of the study. Amphetamine was the most commonly reported lifetime drug of choice in this group, raising the question of whether the initial use of amphetamine was an attempt to self-medicate symptoms of ADHD.

In contrast to most previous treatment studies of adult ADHD, we did not exclude participants with current treatment for comorbid disorders, unless the concurrent treatment interfered with the activity of methylphenidate. This allowed us to extend the external validity of the results to a broader ADHD population. $^{27}$ All participants presented comorbid lifetime substance use disorder and, to the best of our knowledge, this is the first study to establish the efficacy of methylphenidate in adults with ADHD combined with substance use disorder. ${ }^{15}$ Although our findings on the efficacy, effectiveness and safety of methylphenidate treatment of adult $\mathrm{ADHD}$ are consistent with previous studies, ${ }^{15,16,19}$ the effect size of $d=2.17$ was substantially greater than that reported in other studies; for example, previous studies have reported effect sizes ranging from $d=0.42$ to a maximum of $d=1.408 .{ }^{14,15}$ The placebo effect was negligible in this study, in sharp contrast to the significant placebo responses that have been reported in most previous studies. We propose several explanations for these distinctive results. Participants in our study had particularly severe ADHD, leaving a large window for improvement by methylphenidate. In addition to the high baseline ADHD scores, all participants were male and had a

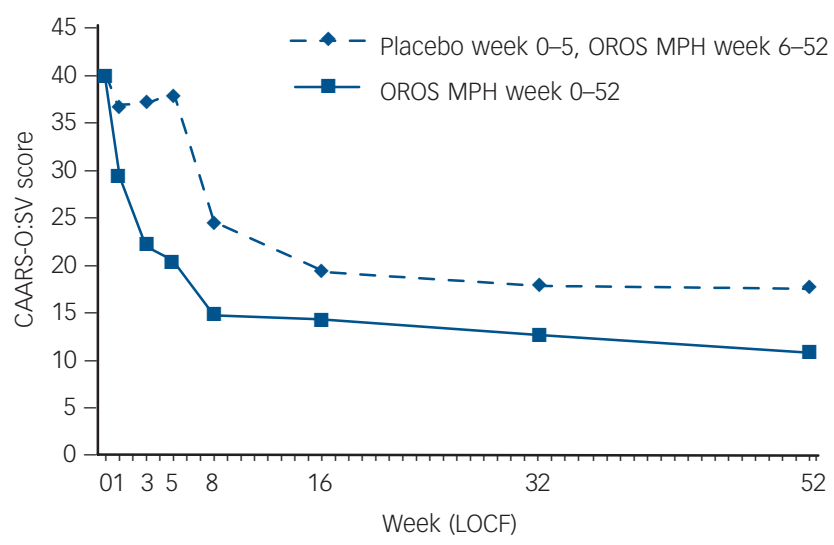

Fig. 2 Intention-to-treat population: Conners' Adult ADHD Rating Scale - Observer: Screening Version (CAARS-O:SV) total sum-score as a function of treatment group and time.

Randomised controlled tria: week 0-5; open-label extension: week 6-52. OROS-MPH, osmotic-release oral system methylphenidate; LOCF, last observation carried forward. history of poor academic achievement. These three factors have been previously suggested as predictors of superior treatment outcomes in adults with ADHD. ${ }^{28}$ The negligible placebo response also contributed to the large treatment effect and we suggest that the high level of ADHD symptoms in the participants also made them less likely to benefit from placebo treatment, consistent with the results of Levin et al. ${ }^{29}$ In our study, diagnostic specificity was likely to be very high due to the comprehensive ADHD assessments that we performed, and this may also have contributed to the large effect size by minimising the number of incorrectly diagnosed study participants. In addition, we achieved excellent treatment adherence, probably due to the structured and controlled setting of the dedicated ADHD wing. This controlled setting may also have contributed to the absence of drug misuse of the study drug. Finally, all participants completed the RCT, thus preserving statistical power.

\section{Limitations and strengths}

The limitations of the present study need to be considered. The sample size was small, although the results were highly significant in the RCT. The open-label extension lacked a control group, and it is difficult to predict how a placebo group may have behaved over the full 52 weeks. The study setting was highly specific, involving long-term male inmates of a high-security prison with ADHD together with comorbid disorders, and generalisation to other populations may not necessarily be valid. However, the inclusion of participants with comorbid disorders with concurrent treatment for these disorders increased the external validity of the results as most adults with ADHD present with comorbid disorders.

\section{Implications}

To our knowledge, this is the first study to evaluate pharmacological treatment of prison inmates with ADHD. Future studies will need to confirm our result in other prison populations. Studies evaluating other pharmacological options and supplementary cognitive-behavioural treatment, and the impact of treatment on relapse into drug misuse and criminality are also warranted. ${ }^{10}$ We propose that future studies of ADHD include comorbid disorders in their inclusion criteria, and that they extend treatment evaluation to include behavioural variables, executive functioning and quality of life.

We conclude by suggesting that OROS methylphenidate can be a viable, effective and safe treatment for ADHD in a prison setting with a well-structured and strictly controlled environment to prevent drug misuse and diversion. Considering the high prevalence of untreated $\mathrm{ADHD}$ in prison inmates, ${ }^{8-10}$ further research on treatment of prison inmates is warranted to confirm and expand upon our findings.

Ylva Ginsberg, MD, Nils Lindefors, MD, PhD, Department of Clinical Neuroscience, Division of Psychiatry and Center of Neurodevelopmental Disorders, Karolinska Institutet, Stockholm, Sweden

Correspondence: Ylva Ginsberg, Psychiatry Southwest, M59, Karolinska University Hospital Huddinge, S-141 86 Stockholm, Sweden. Email: ylva.ginsberg@ki.se

First received 8 Feb 2011, final revision 9 Aug 2011, accepted 15 Sep 2011

\section{Funding}

The Swedish Ministry of Health and Social Affairs and Stockholm County Council, Sweden, funded this study. 


\section{Acknowledgements}

We thank the participants, the staff at Norrtälje Prison and the psychiatrists and psychologists who assisted in the conduct of the trial. We especially acknowledge Michaela Wallensteen, RN (Stockholm County Council) for serving as the masked evaluator, Birgitta Strandberg. RS, BSC (Karolinska Trial Alliance) for monitoring the study, Monica Hellberg for providing administrative assistance and valuable comments on the manuscript.

\section{References}

1 Simon V, Czobor P, Bálint S, Mészáros A, Bitter I. Prevalence and correlates of adult attention-deficit hyperactivity disorder: meta-analysis. Br J Psychiatry 2009; 194: 204-11.

2 American Psychiatric Association. Diagnostic and Statistical Manual of Mental Disorders (4th edn) (DSM-IV). APA, 1994.

3 Biederman J, Mick E, Faraone SV. Age-dependent decline of symptoms of attention deficit hyperactivity disorder: impact of remission definition and symptom type. Am J Psychiatry 2000; 157: 816-8.

4 Kessler RC, Adler L, Ames M, Barkley RA, Birnbaum H, Greenberg P, et al. The prevalence and effects of adult attention deficit/hyperactivity disorder on work performance in a nationally representative sample of workers. J Occup Environ 2005; 47: 565-72.

5 Hammerness $\mathrm{P}$, Surman C, Miller K. Update on adult attention-deficit/ hyperactivity disorder. Curr Neurol Neurosci Rep 2008; 8: 484-9.

6 Rosler M, Casas M, Konofal E, Buitelaar J. Attention deficit hyperactivity disorder in adults. World J Biol Psychiatry 2010; 5: 684-98.

7 Eme RF. Attention-deficit/hyperactivity disorder and correctional health care. J Correct Health Care 2009; 5: 5-18.

8 Ginsberg $\mathrm{Y}$, Hirvikoski T, Lindefors N. Attention deficit hyperactivity disorder (ADHD) among longer-term prison inmates is a prevalent, persistent and disabling disorder. BMC Psychiatry 2010; 10: 112

9 Rosler M, Retz W, Retz-Junginger P, Hengesch G, Schneider M, Supprian T, et al. Prevalence of attention deficit-/hyperactivity disorder (ADHD) and comorbid disorders in young male prison inmates. Eur Arch Psychiatry Clin Neurosci 2004; 254: 365-71.

10 Young SJ, Adamou M, Bolea B, Gudjonsson G, Muller U, Pitts M, et al. The identification and management of ADHD offenders within the criminal justice system: a consensus statment from the UK Adult ADHD Network and criminal justice agencies. BMC Psychiatry 2011; 11: 32.

11 Gudjonsson GH, Wells J, Young S. Personality disorders and clinical syndromes in ADHD prisoners. J Atten Disord 2010; Oct 26 (Epub ahead of print).

12 Young S, Wells J, Gudjonsson G. Predictors of offending among prisoners: the role of attention-deficit hyperactivity disorder and substance use. J Psychopharmacol 2010; Jun 17 (Epub ahead of print).

13 National Institute for Health and Clinical Excellence. Attention Deficit Hyperactivity Disorder. Diagnosis and Management of ADHD in Children, Young People and Adults. NICE, 2009 (http://guidance.nice.org.uk/CG72/ NICEGuidance/).

14 Faraone SV, Spencer T, Aleardi M, Pagano C, Biederman J. Meta-analysis of the efficacy of methylphenidate for treating adult attention-deficit/ hyperactivity disorder. J Clin Psychopharmacol 2004; 24: 24-9.
15 Koesters M, Becker T, Kilian R, Fegert JM, Weinmann S. Limits of metaanalysis: methylphenidate in the treatment of adult attention-deficit hyperactivity disorder. J Psychopharmacol 2009; 23: 733-44.

16 Adler LA, Spencer T, McGough JJ, Jiang H, Muniz R. Long-term effectiveness and safety of dexmethylphenidate extended-release capsules in adult ADHD. J Atten Disord 2009; 12: 449-59.

17 Bejerot S, Ryden EM, Arlinde CM. Two-year outcome of treatment with central stimulant medication in adult attention-deficit/hyperactivity disorder: a prospective study. J Clin Psychiatry 2010; Jun 1 (Epub ahead of print).

18 Rösler M, Fischer R, Ammer R, Ose C, Retz W. A randomised, placebocontrolled, 24-week, study of low-dose extended-release methylphenidate in adults with attention-deficit/hyperactivity disorder. Eur Arch Psychiatry Clin Neurosci 2009; 259: 120-9.

19 Wender PH, Reimherr FW, Marchant BK, Sanford ME, Czajkowski LA, Tomb DA. A one year trial of methylphenidate in the treatment of ADHD. J Atten Disord 2010; 15: 36-45.

20 Lobbestael J, Leurgans M, Arntz A. Inter-rater reliability of the Structured Clinical Interview for DSM-IV Axis I Disorders (SCID I) and Axis II Disorders (SCID II). Clin Psychol Psychother 2010; 18: 75-9.

21 Hare RD, Clark D, Grann M, Thornton D. Psychopathy and the predictive validity of the PCL-R: an international perspective. Behav Sci Law 2000; 18 623-45.

22 Rosler M, Retz W, Stieglitz RD. Psychopathological rating scales as efficacy parameters in adult ADHD treatment investigations - benchmarking instruments for international multicentre trials. Pharmacopsychiatry 2010; 43: 92-8.

23 Guy W. Clinical Global Impressions. US Department of Health and Human Services, 1976.

24 Ramirez A, Ekselius L, Ramklint M. Axis V - Global Assessment of Functioning scale (GAF), further evaluation of the self-report version. Eur Psychiatry 2008; 23: $575-9$.

25 Medori R, Ramos-Quiroga JA, Casas M, Kooij JJ, Niemela A, Trott GE, et al. A randomized, placebo-controlled trial of three fixed dosages of prolonged release OROS methylphenidate in adults with attention-deficit/hyperactivity disorder. Biol Psychiatry 2008; 63: 981-9.

26 Spencer T, Biederman J, Wilens T, Doyle R, Surman C, Prince J, et al. A large, double-blind, randomized clinical trial of methylphenidate in the treatment of adults with attention-deficit/hyperactivity disorder. Biol Psychiatry 2005; 57: 456-63.

27 Surman CB, Monuteaux MC, Petty CR, Faraone SV, Spencer TJ, Chu NF, et al. Representativeness of participants in a clinical trial for attention-deficit/ hyperactivity disorder? Comparison with adults from a large observational study. J Clin Psychiatry 2010; 71: 1612-6.

28 Buitelaar JK, Kooij J, Ramos-Quiroga JA, Dejonckheere J, Casas M, van Oene $\mathrm{JC}$, et al. Predictors of treatment outcome in adults with ADHD treated with OROS methylphenidate. Prog Neuropsychopharmacol Biol Psychiatry 2011; 35: $554-60$.

29 Levin FR, Evans SM, Brooks DJ, Kalbag AS, Garawi F, Nunes EV. Treatment of methadone-maintained patients with adult ADHD: double-blind comparison of methylphenidate, bupropion and placebo. Drug Alcohol Depend 2006; 81: $37-48$. 